\title{
Large-Area MXene Electrode Array for Flexible Electronics
}

Benzheng Lyu, ${ }^{1}$ Minje Kim, ${ }^{1}$ Hongyue Jing, ${ }^{1}$ Joohoon Kang, ${ }^{2}$ Chuan Qian, ${ }^{4}$ Sungjoo Lee, ${ }^{1,3}$ Jeong Ho $\mathrm{Cho}^{4, *}$

${ }^{1}$ SKKU Advanced Institute of Nanotechnology (SAINT), ${ }^{2}$ School of Advanced Materials Science and Engineering, ${ }^{3}$ Department of Nano Engineering, Sungkyunkwan University (SKKU), Suwon 16419, Republic of Korea.

${ }^{4}$ Department of Chemical and Biomolecular Engineering, Yonsei University, Seoul 03722, Republic of Korea.

*Corresponding author: jhcho94@yonsei.ac.kr 
semiconductor

(top: pentacene; bottom: PTCDI- $\mathrm{C}_{8}$ )

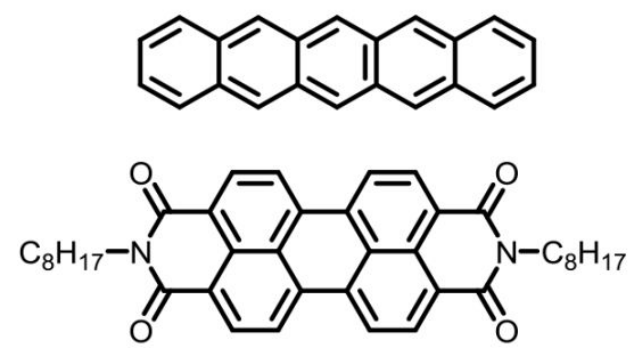

gate dielectric

(PVP)

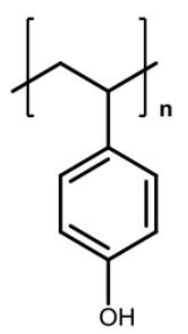

Figure S1. Chemical structures of pentacene, PTCDI- $\mathrm{C}_{8}$, and PVP.

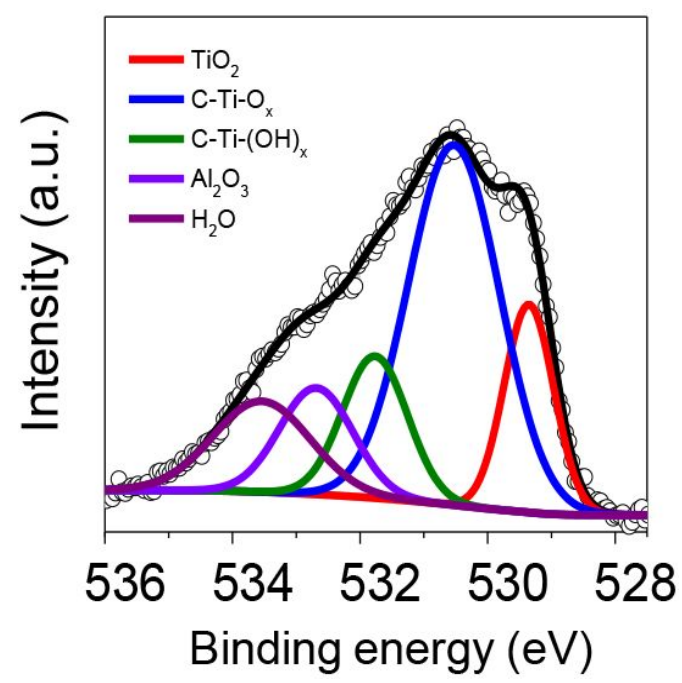

Figure S2. O 1s X-ray photoemission spectroscopy (XPS) spectra of MXene. 

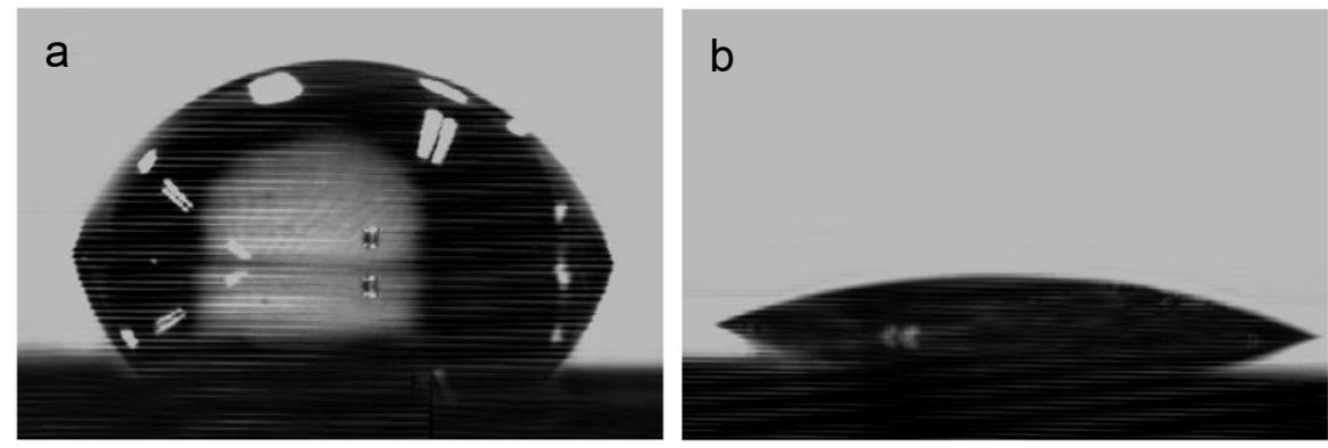

Figure S3. Water contact angle on cPVP surface (a) before and (b) after UV/ozone treatment.
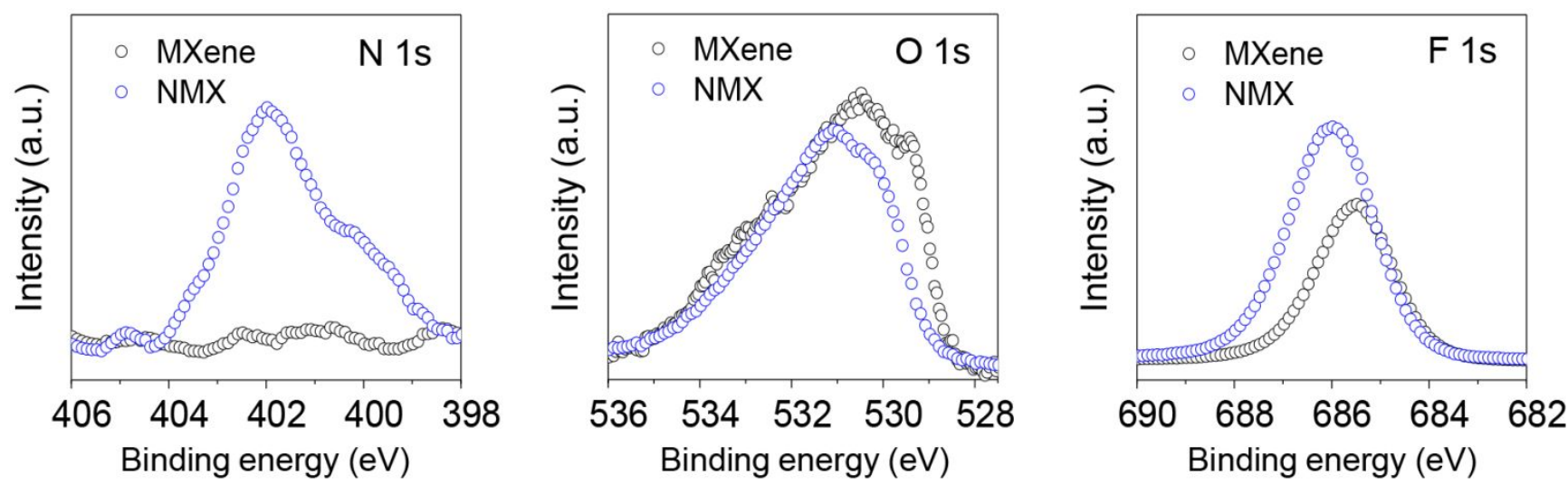

Figure S4. X-ray photoemission spectroscopy (XPS) spectra of MXene and NMX. 


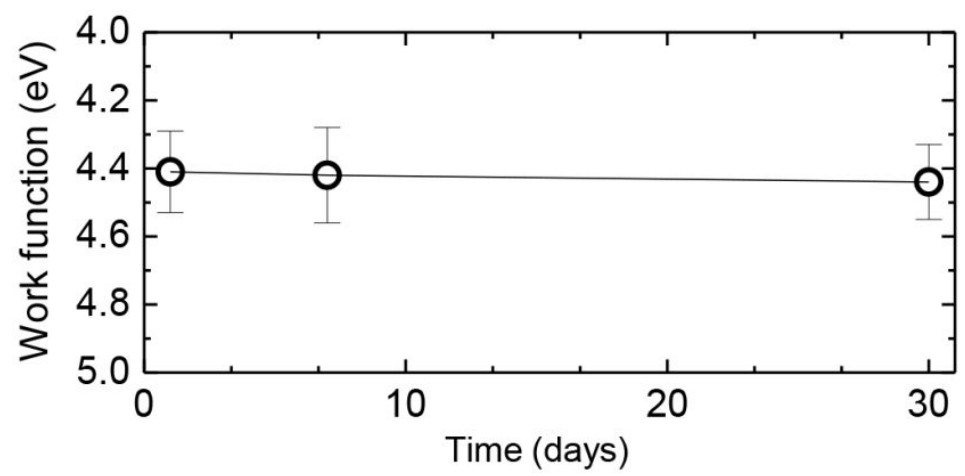

Figure S5. Work function stability of NMX as a function of the storage time in Ar environment.

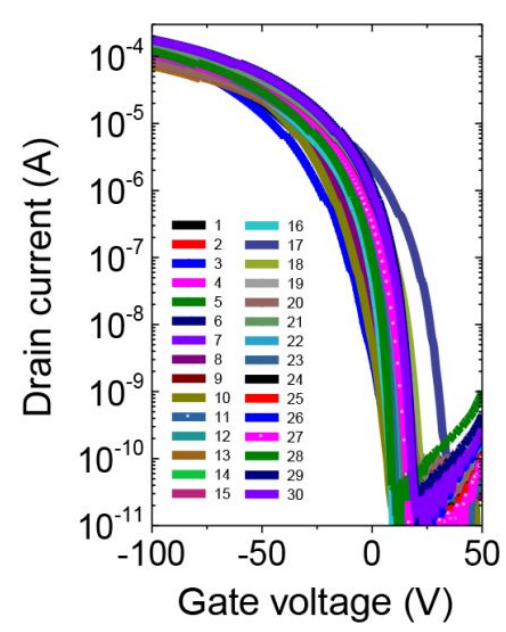

Figure S6. Transfer characteristics of $p$-type pentacene OFETs with pristine $\mathrm{Ti}_{3} \mathrm{C}_{2} \mathrm{~T}_{x}$ MXene electrodes. 


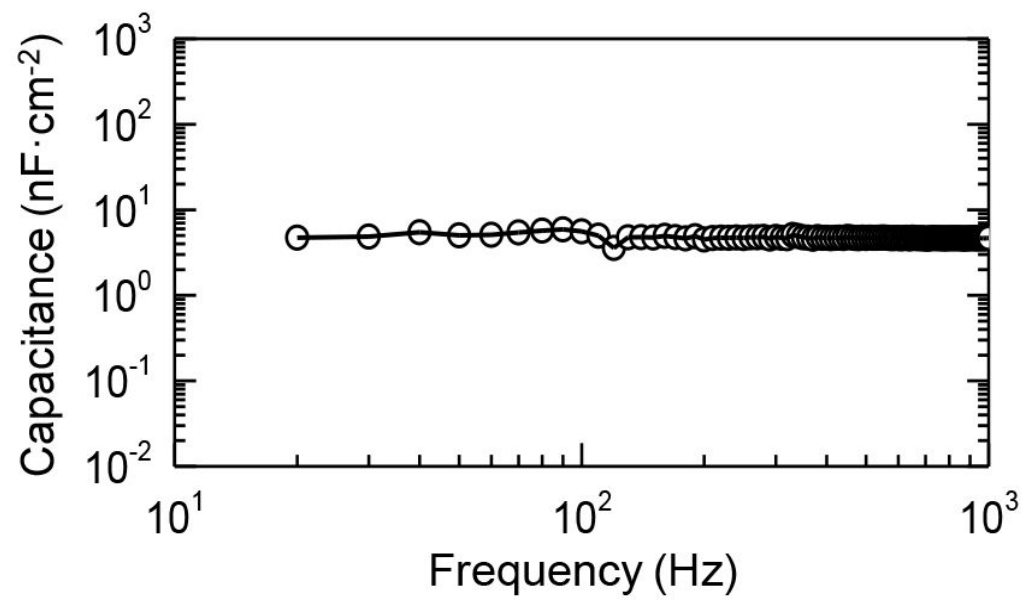

Figure S7. Capacitance values as a function of low frequency.
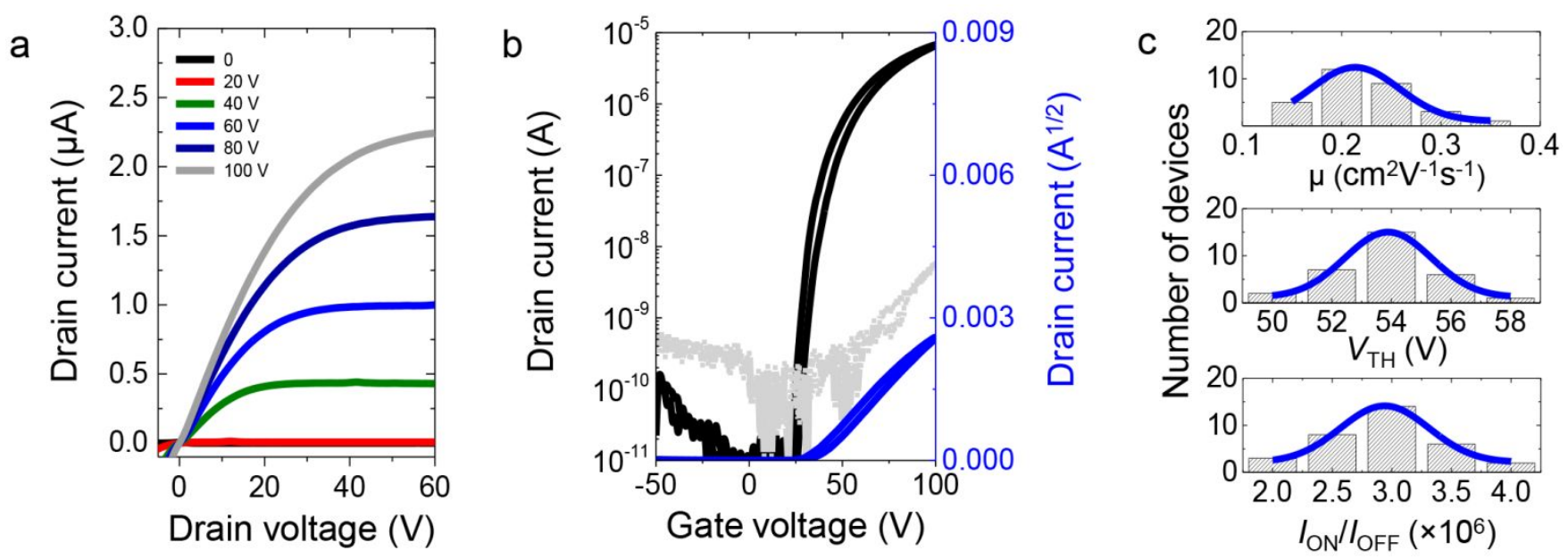

Figure S8. (a) Output characteristics and (b) transfer characteristics of $n$-type PTCDI-C 8 OFETs with pristine MXene electrodes. (c) Histogram and normal distribution of electrical properties (mobility, $V_{\mathrm{TH}}$, and $\left.I_{\mathrm{ON}} / I_{\mathrm{OFF}}\right)$ of $n$-type PTCDI-C 8 OFETs with MXene electrodes. 

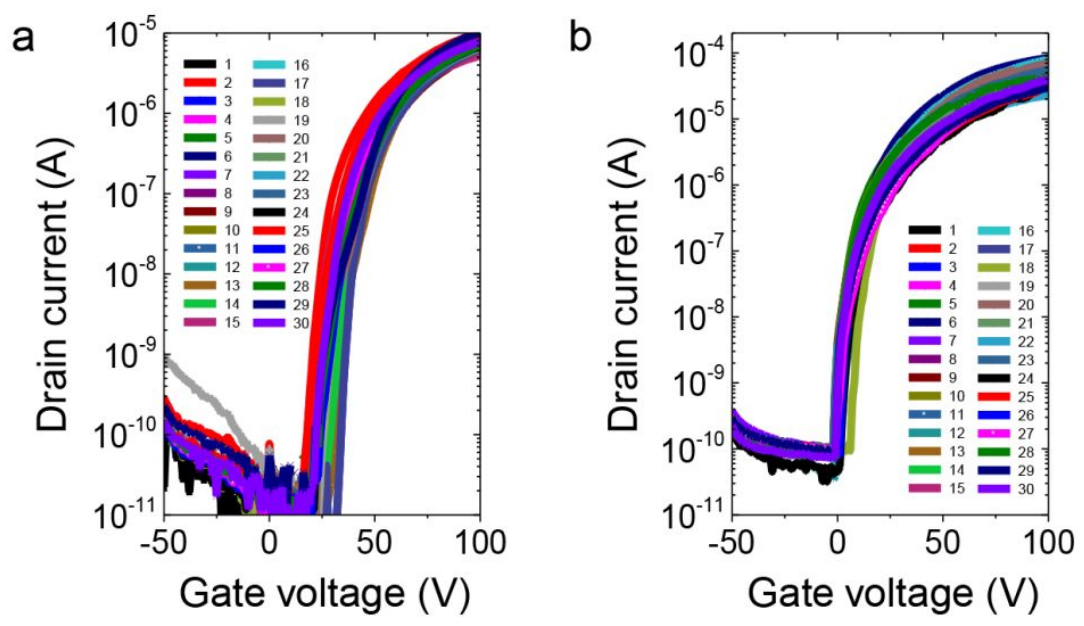

Figure S9. Transfer characteristics of $n$-type PTCDI-C $\mathrm{C}_{8}$ OFETs with (a) pristine $\mathrm{Ti}_{3} \mathrm{C}_{2} \mathrm{~T}_{x}$ MXene and (b) NMX electrodes.
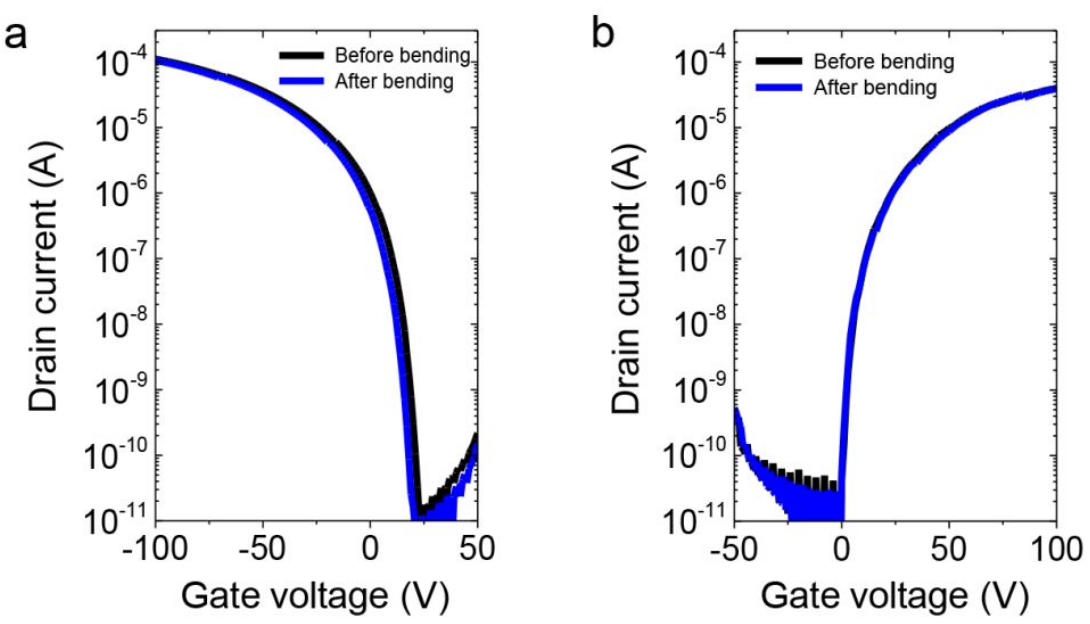

Figure S10. Transfer characteristic of (a) MXene/pentacene OFET and (b) NMX/PTCDI-C ${ }_{8}$ OFET before and after 1000 bending cycles. The bending strain $(S)$ was fixed as $1.05 \%$, according to the following equation: $S=0.5 \cdot\left(t_{\text {sub }}+t_{\text {film }}\right) / R$, where $t_{\text {sub }}$ and $t_{\text {film }}$ are the thicknesses of the substrate $(\sim 125 \mu \mathrm{m})$ and the device $(\sim 540 \mathrm{~nm})$, respectively, and $R$ is the bending radius $(\sim 6 \mathrm{~mm})$. 
a

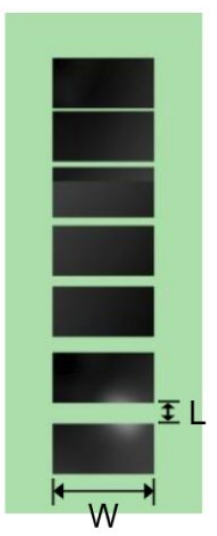

b

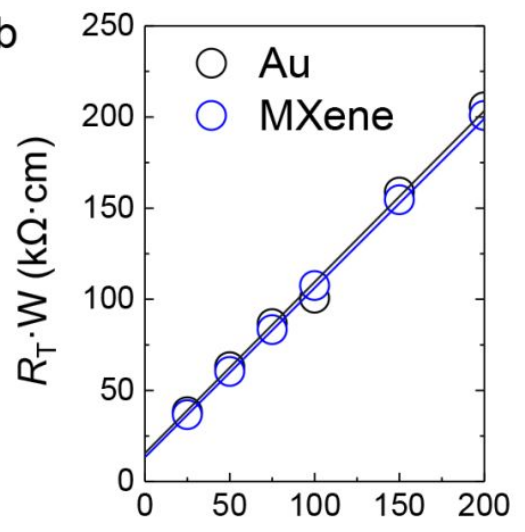

Channel length $(\mu \mathrm{m})$

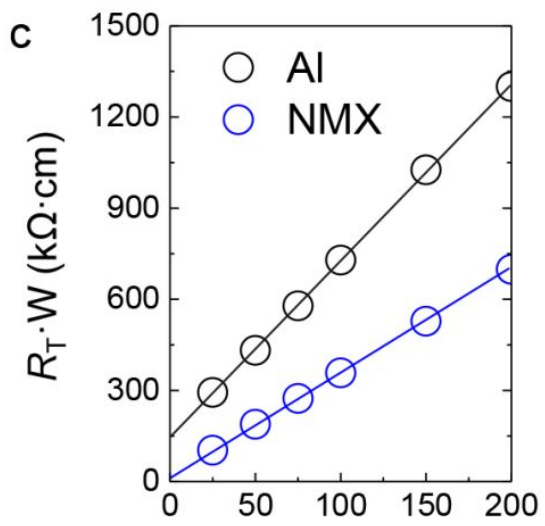

Channel length $(\mu \mathrm{m})$

Figure S11. (a) Transmission-line method (TLM) patterns ( $W=1000 \mu \mathrm{m}, L=25,50,75,100,150,200$ $\mu \mathrm{m})$. (b-c) Width normalized total resistance $\left(R_{\mathrm{T}} \cdot W\right)$ for $(\mathrm{b})$ pentacene OFETs $\left(V_{\mathrm{G}}=-60 \mathrm{~V}\right)$ and (c) PTCDI-C 8 OFETs $\left(V_{\mathrm{G}}=+60 \mathrm{~V}\right)$. The contact resistance $\left(R_{\mathrm{C}}\right)$ was calculated according to the equation of $R_{\mathrm{T}} \cdot W=R_{\mathrm{S}} \cdot L+2 R_{\mathrm{C}} \cdot W$, where $R_{\mathrm{S}}$ is the channel resistance.
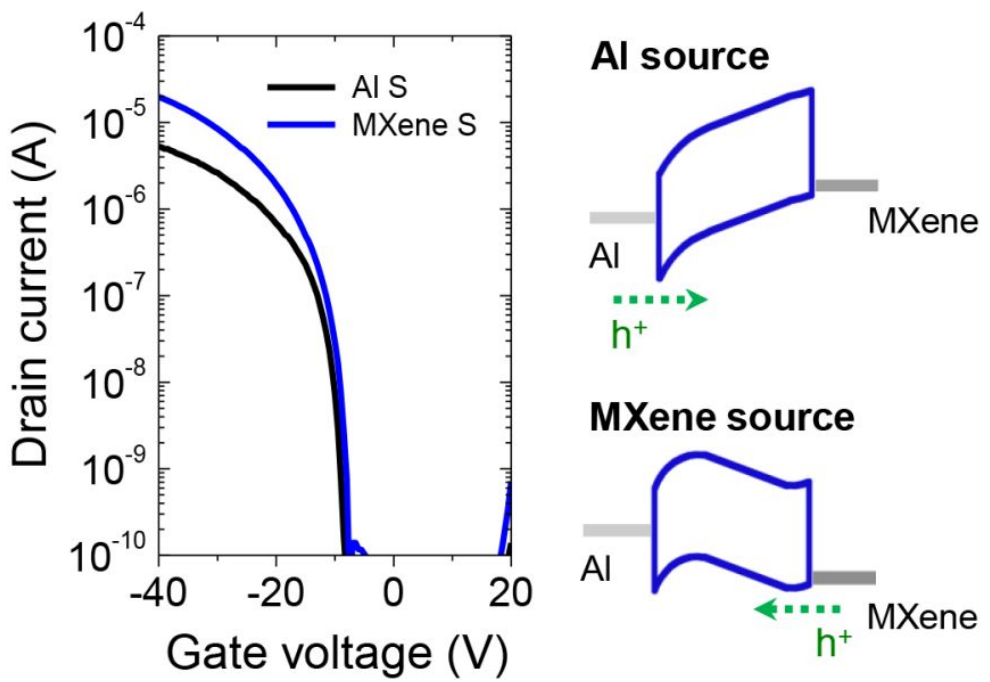

Figure S12. Transfer characteristics of $p$-type pentacene OFETs with Al source electrode (black) and MXene source electrode (blue). The right panel shows schematic band diagrams that illustrate the operation of OFETs with Al source electrode (upper) and MXene source electrode (lower). 

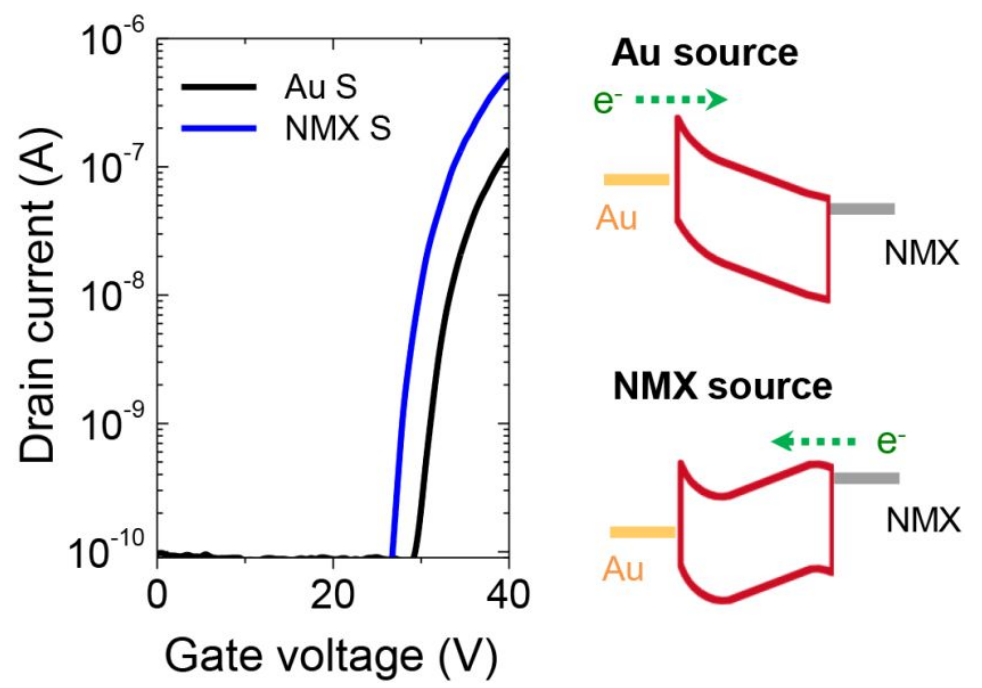

NMX source

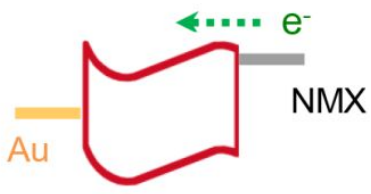

Figure S13. Transfer characteristics of $n$-type PTCDI- $\mathrm{C}_{8}$ OFETs with Au source electrode (black) and MXene source electrode (blue). The right panel shows schematic band diagrams that illustrate the operation of OFETs with Au source electrode (upper) and MXene source electrode (lower). 